\title{
Plasma Citrulline: A New Marker of Gut Epithelium Alteration in Obese Patients?
}

\author{
Stefano Benedini1,2*, Isabella Fermo ${ }^{3}$, Andrea Caumo', Ileana Terruzzi' ${ }^{4}$, Livio Luzi ${ }^{1,2}$ \\ ${ }^{1}$ Department of Biomedical Sciences and Health, Università degli Studi di Milano, Milan, Italy \\ ${ }^{2}$ Metabolism Research Center, IRCCS Policlinico San Donato, Milan, Italy \\ ${ }^{3}$ Division of Immunology, Transplantation \& Infectious disease San Raffaele Scientific Institute, Milan, Italy \\ ${ }^{4}$ Diabetes Research Institute, Metabolism, Nutrigenomics and Cellular Differentiation Unit, San Raffaele \\ Scientific Institute, Milan, Italy \\ Email: "stefano.benedini@unimi.it
}

Received 10 August 2015; accepted 25 October 2015; published 28 October 2015

Copyright (C) 2015 by authors and Scientific Research Publishing Inc.

This work is licensed under the Creative Commons Attribution International License (CC BY).

http://creativecommons.org/licenses/by/4.0/

c) (i) Open Access

\section{Abstract}

Objectives: In the last decade gut microbial diversity was associated with the pathogenesis of obesity in humans. Plasma citrulline was a simple and accurate biomarker for the severity of intestinal failure and was associated with short bowel syndrome and alteration of gut permeability, being developed as an alternative to D-xylose tolerance test for the diagnosis of an abnormal small intestinal absorption of nutrients. This study was performed to ascertain whether obesity might be associated with dysregulation of epithelial gut function. Methods: Fifteen obese individuals (5 $\mathrm{M} / 10 \mathrm{~F}$; BMI $37.4 \pm 6.1 \mathrm{Kg} / \mathrm{m}^{2} ; 42 \pm 6 \mathrm{yrs}$ ) and 15 healthy gender- and age-matched controls (6 M/9 F BMI: $22.7 \pm 2.1 \mathrm{Kg} / \mathrm{m}^{2} ; 39 \pm 7 \mathrm{yrs}$ ) underwent D-xylose load (25 g) and plasma citrulline, plasma insulin, glucose and lipid profile testing. Results: Plasma citrulline was significantly lower in the obese group $(p=0.045)$ with respect to controls, whilst total cholesterol, LDL and tryglicerides concentration, insulin level and HOMA-IR were significantly higher in obese patients. In contrast, after D-xylose load no difference in serum xylose was found between the two groups ( $p=n s)$. Conclusions: Obese patients show a decreased citrulline concentration with respect to lean subjects. Since citrulline is a known marker of intestinal health, alterations in the gut epithelium are likely to be associated with the obesity syndrome. We propose to measure citrulline level in obese patients on a routine basis.

\section{Keywords}

Citrulline, Obesity, HOMA-IR, Gut

\footnotetext{
${ }^{*}$ Corresponding author.
} 


\section{Introduction}

Overweight and obesity are major risk factors for a number of chronic-degenerative diseases. In the last decade gut microbial diversity was associated to the pathogenesis of obesity in humans [1] [2]. It has been previously shown that in patients with short bowel syndrome, plasma citrulline is a biomarker for the severity of intestinal failure [3]. Citrulline is an amino acid released exclusively from small bowel enterocytes and its blood level is highly dependent on small bowel enterocytes mass. In several intestinal diseases there is an alteration of microflora which can play an important role in initiation of the disease. Another methodology to assess gut physiology is D-xylose test. The D-xylose test is an excellent serologic test for the diagnosis of important inflammatory intestinal conditions such as celiac disease [4]. In contrast, plasma citrulline concentration is a simple and reliable surrogate for small bowel absorptive capacity and is not influenced by intestinal inflammation.

\section{Subjects and Methods}

\subsection{Subjects}

Fifteen obese patients without diabetes mellitus, dyslipidemia and in stable condition were recruited in the population of over 600 patients at the Nutrition/Metabolism Unit of San Raffaele Hospital. A BMI of $\geq 30 \mathrm{~kg} / \mathrm{m}^{2}$ was used for obesity classification. Fifteen healthy volunteers, matched for anthropometric parameters, served as a control group. All the subjects were recruited between July 2007 and January 2008. Table 1 represents the anthropometry of study-subjects. The Ethics Committee of San Raphael Scientific Institute (Milan, Italy) approved the experimental protocol, and written informed consent was obtained from all patients.

\subsection{Experimental Protocol}

All subjects involved in this protocol were studied on two separate days. The subjects assumed an isocaloric diet in the 4 weeks preceding the study. On day 1, they were admitted to the Metabolic Unit of the Department of Medicine of San Rafael Scientific Institute after an overnight fast. At 08.00 of day 1, a polyethylene catheter was inserted in the antecubital vein of one forearm. At 08.30, a basal blood sample for the measurement of hormones and metabolites was drawn and the urinary cortisol concentration was measured in the total urine specimen collected over the preceding 24 hours. Then the patients were discharged. On day 2, all subjects were readmitted to the San Raffaele Hospital and underwent a D-xylose load (25 g) as well a blood sample for measurement of plasma citrulline concentration.

\subsection{Analytical Methods}

Aliquots of blood for the measurement of metabolites (glucose, triglycerides, total cholesterol, HDL cholesterol, LDL cholesterol, TSH) level were placed in heparinized tubes. Plasma glucose was measured utilizing the glucose oxidase methodology. Blood aliquots for insulin and TSH were collected in tubes for serum separation. Urine collection $(24 \mathrm{~h}$ ) was performed to quantify cortisol excretion. All blood samples were placed in ice until the plasma/serum was prepared by centrifugation at $4^{\circ} \mathrm{C}$ (within $1.5 \mathrm{~h}$ of sampling). All plasma and serum aliquots were frozen at $-60^{\circ} \mathrm{C}$ until later analysis.

\subsection{D-Xylose Test}

All subjects ingested an oral solution composed by $25 \mathrm{~g}$ of D-xylose in $250 \mathrm{ml}$ of water. Blood samples were drawn and measured for D-xylose concentration at baseline and at 120 minutes after xylose ingestion.

\section{Table 1. Anthropometrical and clinical characteristics of the subjects.}

\begin{tabular}{|c|c|c|}
\hline Subject characteristics & Obese patients & Controls \\
\hline $\operatorname{Sex}(M / F)$ & $5 / 10$ & $6 / 9$ \\
\hline Age (y) & $42 \pm 6$ & $39 \pm 7$ \\
\hline Weight (kg) & $97 \pm 22^{*}$ & $69 \pm 15$ \\
\hline Height $(\mathrm{cm})$ & $160 \pm 8$ & $165 \pm 9$ \\
\hline BMI $\left(\mathrm{kg} / \mathrm{m}^{2}\right)$ & $37.4 \pm 6.1^{*}$ & $22.7 \pm 2.1$ \\
\hline
\end{tabular}

${ }^{*} p<0.001$ vs. CON. 


\subsection{Citrulline Determination}

Plasma levels of the amino acid were obtained using an adaptation of an HPLC procedure based on pre-column derivatization with o-phthaldialdehyde (OPA) and carried out on reversed-phase C18 column [5].

\subsection{Statistical Analysis}

All data are expressed as means \pm SD. Comparisons between obese and control groups were performed with the Student's $t$-test for unpaired data (a value of less than 0.05 was considered statistically significant). We used non parametric methods (Mann-Whitney test) to compare HOMA-IR in the groups because the distribution of these dependent variable were not normal.

\section{Results (See Table 2)}

\subsection{D-Xylose Test}

No difference was observed in the xylosemia between the two study groups. In particular the obese group had a value of xylosemia slightly lower compared to controls.

\subsection{Insulin Action}

The HOMA-IR was higher in obese group with respect to controls $(p<0.03)$.

\subsection{Plasma Citrulline}

Plasma citrulline concentration was lower in obese patients with respect to controls $(p=0.045)$, indicating a reduction of production of this amino-acid by small bowel epithelium.

\subsection{Lipid Profile}

Plasma lipid profile was normal in obese patients and in control group. The total cholesterol was higher in obese group $(p<0.01)$. Also triglyceride concentrations were higher in obese group $(p<0.01)$.

\subsection{Hormones}

Plasma insulin was higher in the obese group with respect to controls $(p<0.01)$. The free urinary cortisol was normal in obese patients but within the upper $50 \%$ of the normal range $(p=0.07)$.

Table 2. Mean + SD of lipids, hormones, insulin sensitivity index, plasma citrulline and xylose in obese patients and in control subjects.

\begin{tabular}{ccc}
\hline & Obese patients & Controls \\
\hline Plasma glucose (mmol/l) & $4.7 \pm 0.6$ & $5 \pm 0.3$ \\
Free insulin (pmol/l) & $49.8^{*} \pm 15.06$ & $30.5 \pm 10.1$ \\
Total cholesterol (mmol/l) & $5.2^{*} \pm 0.4$ & $4.4 \pm 0.3$ \\
Cholesterol HDL & $1.3 \pm 0.3$ & $1.4 \pm 0.2$ \\
Cholesterol LDL (calculated) & $3.4^{*} \pm 1.4$ & $2.6 \pm 1.1$ \\
Triglycerides (mmol/l) & $1.3^{*} \pm 0.9$ & $0.7 \pm 0.4$ \\
HOMA-IR & $1.75^{*} \pm 0.93$ & $0.98 \pm 0.44$ \\
Blood D-xylose (mmol/L) & $2.82 \pm 0.76$ & $3.21 \pm 0.89$ \\
Plasma citrulline (mmol/L) & $22.3^{*} \pm 6.2$ & $26.3 \pm 3.9$ \\
Free urinary cortisol (nmol/24 h) & $342 \pm 74$ & $296 \pm 63$ \\
TSH (mU/l) & $1.72 \pm 0.90$ & $1.92 \pm 1.03$ \\
\hline
\end{tabular}

\footnotetext{
${ }_{p}^{*}<0.05$ vs. CON.
} 


\section{Discussion}

The current study presents a new observation regarding the possibility that obesity may be associated with dysregulation of epithelial gut function.

It is well known that intestinal microflora and diet influence both body weight and insulin-resistance, notably through an action on adipose cells [6]. Indeed, it has been suggested that a person's gut microbiota has a specific metabolic efficiency and that certain characteristics of the microbiota composition might predispose to obesity [2].

The gut epithelium is not only involved in the absorption of nutrients, but must also provide a barrier between the gastrointestinal lumen and the rest of the body.

In our study, all obese subjects were insulin resistant and with alteration of gut epithelium, possibly of inflammatory origin.

In patients with intestinal transplantation low value of blood citrulline is now considered a marker of graft dysfunction (acute rejection) [7].

In animal model citrulline supplementation improved gut function by decreasing intestinal permeability and positively affecting immune function [8].

In the same fashion the alterations present in obese patients seem to be similar to patients with early stage of graft rejection.

One possible explanation of our results in the obese subjects may be the presence of chronic inflammation in the epithelium of gut.

Therapies of obesity have focused on reducing caloric intake, decreasing nutrient absorption, or increasing physical activity. Future treatments for obesity may attempt to modulate the intestinal microbiota through the use of antibiotics, probiotics, and prebiotics [9].

\section{Conclusions and Limitations}

In conclusion, our results indicate that there is a strict correlation between the characteristic metabolic picture present in obesity and low value of citrulline (a marker of integrity of gut epithelium). In accordance with other authors [10] [11], we showed in humans the presence of epithelium gut alteration in obesity.

There are some possible limitations to consider in the present study. First, the number of obesity in the sample is somewhat small. Second, this is a case-control study and we cannot draw a cause-effect conclusion about gastrointestinal alteration and obesity.

Further studies are needed to elucidate the potential role of citrulline as useful biomarker to label patients affected by obesity as patients with dysregulation of epithelial gut absorption.

\section{Acknowledgements}

This study was fully supported by a grant kindly provided by BRACCO, SpA.

\section{References}

[1] DiBaise, J.K., Zhang, H., Crowell, M.D., Krajmalnik-Brown, R., Decker, G.A. and Rittmann, B.E. (2008) Gut Microbiota and Its Possible Relationship with Obesity. Mayo Clinic Proceedings, 83, 460-469. http://dx.doi.org/10.4065/83.4.460

[2] Backhed, F., Ley, R.E., Sonnenburg, J.L., Peterson, D.A. and Gordon, J.I. (2005) Host-Bacterial Mutualism in the Human Intestine. Science, 307, 1915-1920. http://dx.doi.org/10.1126/science.1104816

[3] Gong, J.F., Zhu, W.M., Li, N., Liu, F.N., Tan, L., Luo, N. and Li, J.S. (2005) Serum Citrulline Is a Simple Quantitative Marker for Small Intestinal Enterocytes Mass and Absorption Function in Short Bowel Patients. Journal of Surgical Research, 127, 177-182. http://dx.doi.org/10.1016/j.jss.2005.04.004

[4] Ehrenpreis, E.D., Salvino, M. and Craig, R.M. (2001) Improving the Serum D-Xylose Test for the Identification of Patients with Small Intestinal Malabsorption. Journal of Clinical Gastroenterology, 33, 36-40. http://dx.doi.org/10.1097/00004836-200107000-00009

[5] Fermo, I., De Vecchi, E., Diomede, L. and Paroni, R. (1990) Serum Amino Acid Analysis with Pre-Column Derivatization: Comparison of the o-Phthaldialdehyde and N,N-Diethyl-2,4-dinitro-5-fluoroaniline Methods. Journal of Chromatography B, 534, 23-35. http://dx.doi.org/10.1016/S0378-4347(00)82145-3

[6] Turnbaugh, P.J., Ley, R.E., Mahowald, M.A., Magrini, V., Mardis, E.R. and Gordon, J.I. (2006) An Obesity-Asso- 
ciated Gut Microbiome with Increased Capacity for Energy Harvest. Nature, 444, 1027-1031.

[7] David, A.I., Selvaggi, G., Ruiz, P., Gaynor, J.J., Tryphonopoulos, P., Kleiner, G.I., Moon, J.I., Nishida, S., Pappas, P.A., Conanan, L., Weppler, D., Esquenazi, V., Levi, D.M., Kato, T. and Tzakis, A.G. (2007) Blood Citrulline Level Is an Exclusionary Marker for Significant Acute Rejection after Intestinal Transplantation. Transplantation, 84, 1077 1081.

[8] Batista, M.A., Nicoli, J.R., Martins, F.S., Machado, J.A.N., Arantes, R.M.E., Quirino, I.E.P., et al. (2012) Pretreatment with Citrulline Improves Gut Barrier after Intestinal Obstruction in Mice. Journal of Parenteral and Enteral Nutrition, 36, 69-76. http://dx.doi.org/10.1177/0148607111414024

[9] Tennyson, C.A. and Friedman, G. (2008) Microecology, Obesity, and Probiotics. Current Opinion in Endocrinology, Diabetes \& Obesity, 15, 422-427. http://dx.doi.org/10.1097/MED.0b013e328308dbfb

[10] Brun, P., Castagliuolo, I., Di Leo, V., Buda, A., Pinzani, M., Palu, G. and Martines, D. (2007) Increased Intestinal Permeability in Obese Mice: New Evidence in the Pathogenesis of Nonalcoholic Steatohepatitis. American Journal of Physiology-Gastrointestinal and Liver Physiology, 292, G518-G525. http://dx.doi.org/10.1152/ajpgi.00024.2006

[11] Osbak, P.S., Bindslev, N. and Hansen, M.B. (2011) Relationships between Body Mass Index and Short-Circuit Current in Human Duodenal and Colonic Mucosal Biopsies. Acta Physiologica (Oxford), 201, 47-53. http://dx.doi.org/10.1111/j.1748-1716.2010.02202.x 